\title{
Effet de la saison sur la transpiration de feuilles de rosiers cv Sonia, cultivés en hors-sol, à 2 niveaux d'électroconductivité et d'humidité relative
}

\author{
L Urban*, P Pyrrha, G Perez \\ INRA, unité de recherche intégrée en norticulture, route des Colles, \\ Sophia-Antipolis, F06410 Biot, France
}

(Reçu le 3 juin 1993 ; accepté le 6 décembre 1993)

\begin{abstract}
Résumé - Des plants de rosier (Rosa hybrida cv Sonia) ont été cultivés en laine de roche dans 2 serres chauffées : une serre témoin (humidité relative (HR) basse) et une serre équipée d'un dispositif de régulation de I'HR (HR élevée, supérieure à $70 \%$ ). Deux niveaux d'électroconductivité (EC, exprimant la salinité) ont été appliqués: un niveau élevé $\left(E C=3,8 \mathrm{mS} \mathrm{cm}^{-1}\right)$, et un niveau bas $\left(E C=1,8 \mathrm{mS} \mathrm{cm}^{-1}\right)$. Dans les 2 cas, le taux de drainage a été maintenu à plus de $40 \%$. Le potentiel hydrique à la fermeture stomatique complète $\left(\Psi^{\text {fer }}\right)$ et la transpiration cuticulaire $\left(E_{\text {cut }}\right)$ ont été mesurés à 5 périodes différentes au cours d'une année sur des feuilles phénologiquement identiques. $\Psi^{\text {fer }}$ n'a pas été affecté significativement par l'HR ni par l'EC. $\Psi^{\text {fer }}$ a augmenté depuis le printemps jusqu'au début de l'automne et diminué après. Ces variations ne semblent pas correspondre à une adaptation à la sécheresse, ni à un changement ontogénétique. $E_{c u t}$ a chuté fortement au début de l'automne. Les niveaux de $E_{\text {cut }}$ les plus bas ont été observés dans la serre à $\mathrm{HR}$ basse. Les causes de ces différences et variations sont discutées.
\end{abstract}

culture hydroponique / rosier / serre / transpiration / variation saisonnière

Summary - Effect of season on the transpiration of Sonia rose leaves grown in rockwool at 2 different levels of electrical conductivity and relative humidity. Rose plants (Rosa hybrida cv Sonia) were grown in rockwool in 2 heated greenhouses: 1 control (referred as the low relative humidity (RH) greenhouse) and 1 with $\mathrm{RH}$ control (high $R H$ greenhouse). Two electrical conductivity (EC, expressing salinity) rates were applied: high (EC of drainage water around $3.8 \mathrm{mS} \mathrm{cm}^{-1}$ ), and low (EC of drainage water around $1.8 \mathrm{mS} \mathrm{cm}^{-1}$ ). In both cases, the drainage rate was kept above $40 \%$ of the supplied water. The water potential at stomatal closure $\left(\psi^{\text {fer }}\right)$ and the cuticular transpiration rate $\left(E_{c u t}\right)$ were measured throughout the season on phenologically identical leaves. $\psi^{\text {fer }}$ was not affected by RH or EC. It increased from spring to early fall, recovering partly from early fall to winter. These variations do not seem to result from a water stress adaptative process or ontogenetic changes taking place in the leaves. Ecut decreased dramatically in early fall. It was the lowest in the low RH greenhouse. These differences and fluctuations are discussed.

greenhouse cultivation / Rosa hybrida / seasonal variations / soilless cultivation / transpiration rate

\section{INTRODUCTION}

Les relations hydriques jouent un rôle fondamental dans la durée de vie en vase des fleurs coupées. Ainsi, les séjours à sec favorisent la dégradation du bilan hydrique des fleurs coupées et tendent à hâter l'apparition des premiers symptômes de sénescence (Masson et Paulin, 1980 ; Coker et al, 1985 ;

\footnotetext{
* Correspondances et tirés à part
} 
Paulin et al, 1985). Inversement, l'application d'antitranspirants a permis d'augmenter la durée de vie en vase de fleurs d'Anthurium scherzerianum Schott en favorisant le maintien d'un bilan hydrique positif (Paull et Goo, 1985).

Dans le cas des roses, les feuilles représentent le siège principal de la transpiration. En effet, les pétales du bouton floral sont fortement imbriqués les uns dans les autres, au moins jusqu'au stade de plein épanouissement. Leur surface évaporante est donc faible par rapport à celle des feuilles qui sont, elles, au contraire, bien étalées. II faut $3 \mathrm{j}$ environ pour déshydrater jusqu'à $70 \%$ de teneur en eau relative un bouton floral au stade de récolte, alors qu'il suffit généralement de moins de $7 \mathrm{~h}$ pour arriver au même niveau de déshydratation avec une feuille (résultats non publiés).

Dans un contexte climatique donné, la transpiration foliaire dépend principalement de l'ouverture des stomates et, secondairement, de la résistance à la diffusion de la vapeur d'eau de la cuticule. La transpiration cuticulaire représente en général une fraction négligeable de la transpiration foliaire lorsque les stomates sont bien ouverts. Mais, pour les feuilles de tiges florales coupées, placées en conditions de faible éclairement, les stomates sont moins ouverts qu'en conditions naturelles. De plus, le poids relatif de la cuticule dans la transpiration foliaire augmente après les premiers jours de vie en vase. En effet, la transpiration diurne diminue d'un facteur 3 entre les premiers et les derniers jours de vie en vase (Fabret, 1993), indiquant une réduction progressive de l'ouverture des stomates pendant le jour.

Quant à la limitation que les stomates imposent à la perte d'eau par transpiration, elle est d'autant plus importante que leur fermeture intervient plus précocement, c'est-à-dire que la perte d'eau requise avant la fermeture des stomates est faible. Des études menées sur plantes entières ont montré le découplage existant entre l'état hydrique moyen et la conductance stomatique des feuilles de rosiers cultivés en hors-sol avec un arrosage non-limitant (Urban et Langelez, 1992). Tardieu et Davies (1992) ont montré sur épiderme de Commelina communis que la régulation de la conductance stomatique dépendait à la fois de l'apport d'acide abscissique et du potentiel hydrique. Ces observations tendent à réconcilier les points de vue des tenants de la régulation chimique et de ceux de la régulation hydraulique de la conductance stomatique. L'idée que la conductance stomatique dépend du niveau de déshydratation des feuilles reste pertinente dans le cas de feuilles excisées ou rattachées à des tiges florales coupées, qui ne sont donc plus soumises à l'influence de signaux provenant des racines.

L'influence des variations climatiques saisonnières et des conditions de culture sur la sensibilité de la conductance stomatique à la perte d'eau et sur la résistance à la diffusion de la vapeur d'eau de la cuticule a été très peu étudiée. L'objectif de cette étude est de combler cette lacune en analysant l'effet saisonnier sur la valeur de potentiel hydrique correspondant au seuil de fermeture stomatique complète et sur la transpiration cuticulaire de feuilles excisées provenant de plants de rosier cultivés sous serre, en hors-sol, à 2 niveaux de salinité et à 2 niveaux d'humidité relative (HR) pertinents dans un contexte de production.

\section{MATÉRIEL ET MÉTHODES}

Les essais ont été menés pendant la période comprise entre mai 1991 et février 1992. Le matériel végétal était constitué de plants de rosier cv Sonia, obtenus à partir de boutures, mises à enraciner le 2 avril 1990 dans des cubes de laine de roche, puis plantées le 10 mai 1990 en «pains de laine de roche" (Cultilène) de $1 \mathrm{~m}$ de long, $0,2 \mathrm{~m}$ de large et $0,075 \mathrm{~m}$ d'épaisseur, à une densité de 7 plants par pain (densité brute: 5,6 plants par $\mathrm{m}^{2}$ ). La plantation a été effectuée dans 2 serres: l'une équipée d'un dispositif de nébulisation fine (The Fog System), correspondant au traitement à HR élevée, et l'autre d'un écran d'ombrage (Ludwig Svensson LS 16), correspondant au traitement à HR basse.

La température de l'air était maîtrisée dans les 2 serres à l'aide d'un "ordinateur de serre" de marque Seria, équipé de ses propres sondes de température et d'hygrométrie. La température de consigne d'ouverture des "ouvrants" était de $22^{\circ} \mathrm{C}$, celle de chauffage était de $17^{\circ} \mathrm{C}$ minimum. Ces consignes ont été maintenues tout au long de l'essai. La lourdeur du dispositif expérimental ne nous a pas permis de répéter l'essai. En revanche, les paramètres climatiques de chacune des serres ont été enregistrés de façon indépendante, en continu, à l'aide de systèmes d'acquisition de données (Delta Logger de chez Delta T Device), équipés de thermocouples, de psychro- 
mètres artisanaux et de sondes de rayonnement global. Les valeurs moyennes diurnes de température d'air, de déficit de saturation en vapeur d'eau de l'air (DSA) et de rayonnement global cumulé sont fournies, pour chaque serre, dans le tableau I, qui montre que celles-ci se différenciaient par le DSA, mais très peu par la température et le rayonnement reçu.

Dans la serre équipée de l'écran (HR basse), l'ombrage était déclenché lorsque le rayonnement global extérieur atteignait $800 \mathrm{~W} \mathrm{~m} \mathrm{~m}^{-2}$. Dans la serre équipée du dispositif de nébulisation fine (HR élevée), la brumisation était déclenchée automatiquement lorsqu'HR tombait en dessous de $70 \%$. Le système de nébulisation utilisé produit des gouttelettes de moins de $10 \mu \mathrm{m}$ de diamètre, à un débit de $1,9410^{-6} \mathrm{~m}^{3}$ d'eau $\mathrm{s}^{-1} \mathrm{~m}^{-2}$.

La fertilisation suivait les recommandations de Brun et Tramier (1988). La consigne de $\mathrm{pH}$ était fixée à 5,6. L'irrigation fertilisante était déclenchée à l'aide d'un "ordinateur de serre " commercial (Systeem 9, Indal) équipé d'un capteur de rayonnement extérieur de type Kipp en zonen, de sondes de $\mathrm{pH}$ et d'électroconductivité (EC). L'irrigation était déclenchée automatiquement dès que la quantité d'énergie solaire cumulée depuis le dernier arrosage atteignait 2,8 $10^{6} \mathrm{~J} \mathrm{~m}^{-2}$. Chaque irrigation de jour apportait $100 \%$ de l'évapotranspiration potentielle, calculée d'après de Villèle (1972), à partir de la mesure du rayonnement global extérieur. Deux irrigations fertilisantes supplémentaires étaient déclenchées chaque nuit. Dans ces conditions, le drainage cumulé sur $24 \mathrm{~h}$ représentait au moins $40 \%$ de la solution nutritive apportée. L'EC de la solution nutritive apportée était fixée de manière à maintenir environ $0,18 \mathrm{~S}$ $\mathrm{m}^{-1}$ dans le drainage du traitement à salinité basse et $0,38 \mathrm{~S} \mathrm{~m}^{-1}$ dans le drainage du traitement à salinité haute. Les valeurs d'EC de consigne étaient introduites chaque jour dans l'ordinateur de régulation de l'irrigation fertilisante après avoir été calculées sur micro-ordinateur en fonction de données collectées par 12 sondes d'EC (Tacussel XE 100/2.5) et par 12 capteurs de débit de drainage (Honeywell LL 1012000). Le système de mesure et de traitements de données utilisé a été précisément décrit par Burn et al (1994). Le dispositif expérimental était un dispositif factoriel partiellement hiérarchisé comportant 2 serres, 4 blocs à l'intérieur de chaque serre et 2 niveaux d'EC par serre et par bloc.

Les études ont été menées sur 5 périodes : 29 avril-17 mai 1991, 12-27 juin 1991, 15-30 juillet 1991, 16 septembre-1 ${ }^{\text {er }}$ octobre $1991,13-28$ janvier 1992. Une feuille par serre et par EC était prélevée chaque jour dans un bloc donné, pendant 12 j (4 bloc $\times 3$ ) par période considérée, en vue de réaliser des courbes de transpiration. Quatre feuilles ont donc été prélevées chaque jour. Ce nombre correspond au maximum d'échantillons qu'il est possible de traiter simultanément. Les prélèvements étaient effectués à $5 \mathrm{~h}$ pour tenir compte du temps nécessaire à la réalisation des courbes de transpiration (jusqu'à $10 \mathrm{~h}$ ). Les mesures ont toutes porté sur des feuilles jeunes, matures, bien exposées, prélevées sur des tiges florales portant un bouton floral de la taille d'un petit pois au moins. Des mesures préalables avaient permis de montrer que ces feuilles donnaient la réponse la plus homogène. Il est essentiel de souligner que les feuilles utilisées pour ces mesures étaient toutes au même stade phénologique, les rosiers cultivés en serres chauffées produisant des tiges et des feuilles nouvelles tout au long de l'année.

La transpiration foliaire est habituellement étudiée à l'aide de poromètres à diffusion. Ces appareils conviennent bien aux mesures de résistance stomatique. En revanche, leur précision est insuffisante aux faibles niveaux de transpiration (Van Gardinen et Grace, 1992). C'est la raison pour laquelle nous avons choisi d'utiliser la méthode décrite par Hygen $(1951,1953)$, qui permet d'étudier précisément la transpiration cuticulaire de feuilles excisées. Cette méthode repose sur une exploitation des courbes représentant le logarithme du poids d'eau de feuilles, en cours de déshydratation, en fonction

Tableau I. Valeurs moyennes journalières des paramètres climatiques relevés dans la serre-HR basse et dans la serre-HR élevée, par période considérée.

\begin{tabular}{|c|c|c|c|c|c|c|}
\hline \multirow{2}{*}{ Périodes } & \multicolumn{2}{|c|}{$\left.T_{a i r}{ }^{\circ} \mathrm{C}\right)$} & \multicolumn{2}{|c|}{$D S A(k P a)$} & \multicolumn{2}{|c|}{$R g\left(10^{6} J \mathrm{~m}^{-2}\right)$} \\
\hline & $\begin{array}{c}\text { HR } \\
\text { élevée }\end{array}$ & $\begin{array}{c}H R \\
\text { basse }\end{array}$ & $\begin{array}{c}H R \\
\text { élevée }\end{array}$ & $\begin{array}{c}H R \\
\text { basse }\end{array}$ & $\begin{array}{c}\text { HR } \\
\text { élevée }\end{array}$ & $\begin{array}{c}H R \\
\text { basse }\end{array}$ \\
\hline 29 avril-17 mai 1991 & 21,5 & 21,8 & 0,44 & 0,79 & 15,12 & 15,53 \\
\hline 12-27 juin 1991 & 25,6 & 25,1 & 0,48 & 0,87 & 22,03 & 22,42 \\
\hline 15-30 juillet 1991 & 26,1 & 27,4 & 0,89 & 1,33 & 20,55 & 18,80 \\
\hline 16 sept-1 ${ }^{\text {er }}$ oct 1991 & 23,6 & 24,3 & 0,48 & 0,66 & 12,09 & 12,15 \\
\hline 13-28 janvier 1992 & 20,1 & 20,6 & 0,46 & 0,60 & 4,40 & 4,30 \\
\hline
\end{tabular}

$\mathrm{T}_{\text {air }}$ : température de l'air; DSA : déficit de saturation en vapeur d'eau de l'air ; $\mathrm{Rg}$ : quantité de rayonnement global reçue. 
du temps. Le principe de cette méthode peut être résumé de la manière qui suit.

Le taux de transpiration d'un organe excisé, placé en conditions stables, diminue dans le temps en conséquence de la réduction de l'ouverture des stomates et de la diminution de la teneur en eau. Si la diminution de la transpiration en fonction de la teneur en eau d'un organe peut être exprimée par une formule simple, alors toute déviation par rapport à toute courbe théorique, déduite de cette formule, indique un changement dans l'ouverture stomatique.

L'importance et les conditions de validité de ces hypothèses ont été discutées de manière détaillée par Hygen (1951). Celles-ci avaient de bonnes chances d'être vérifiées dans nos essais, dans la mesure où nous avons maintenu des conditions climatiques relativement stables pendant le dessèchement des feuilles $\left(T_{\text {air }}=20 \pm\right.$ $0,5^{\circ} \mathrm{C}, \mathrm{HR}=70 \pm 10 \%$, lumière utile pour la photosynthèse $(\mathrm{PAR})=100 \mu \mathrm{mol} \mathrm{m}^{-2} \mathrm{~s}^{-1}$ ), où la vitesse de dessèchement imposée était lente (généralement moins de $2 \%$ de perte d'eau par heure), et où les nivaux excessifs de dessèchement ont été évités (nous ne sommes jamais descendus en dessous de $55 \%$ de teneur en eau relative).

Dans ces conditions, on peut montrer que le logarithme de la différence (PF-k) décroît proportionnellement avec le temps (avec PF représentant le poids frais et $k$ une constante). Hygen (1951) a proposé plusieurs méthodes pour estimer k. L'une d'entre elles consiste à l'approximer tout simplement par le poids de matière sèche, PS. C'est ce que nous avons fait, représentant donc le logarithme du poids d'eau en fonction du temps (fig 1).

Sur une courbe de transpiration, il est possible de déterminer visuellement le début de la fermeture stomatique et le moment où celle-ci est totale. II est également possible de déterminer, à partir des pentes des droites de la "phase stomatique" et de la "phase cuticulaire", la transpiration maximale (stomatique avec les stomates complètement ouverts + cuticulaire) et la transpiration minimale (cuticulaire uniquement). En fait, nous avons observé que la "phase stomatique" est très courte. La rupture de pente de courbe traduisant le passage de la "phase stomatique " à la "phase de fermeture des stomates" est donc difficile à mettre précisément en évidence chez les feuilles de rosier. En revanche, la détermination du point correspondant à la fin de la "phase de fermeture stomatique » et le calcul de la transpiration cuticulaire $\left(E_{c u t}\right)$ n'ont posé aucune difficulté (fig 1). Nous avons pu vérifier que les coefficients de détermination des régressions provenant de l'ajustement linéaire des points des «phases cuticulaires " des différentes courbes de transpiration obtenues étaient élevés, $r^{2}>0,99$ (avec $P<0,0001$ ). Cela tend à indiquer que la

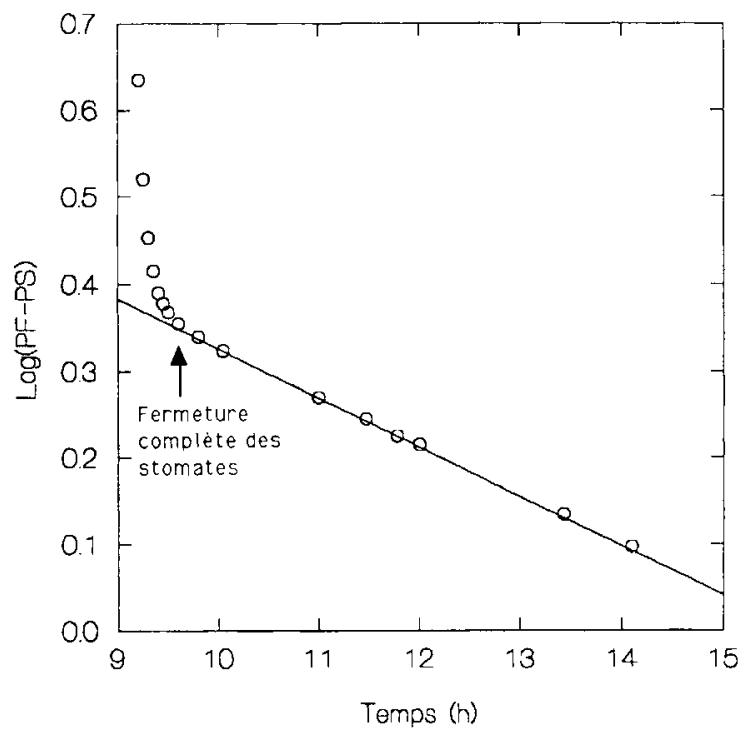

Fig 1. Courbe de transpiration typique (d'après Hygen, 1951), représentant le logarithme de la différence du poids frais et du poids sec d'une feuille de rosier en fonction du temps. Les cercles correspondent à des points réels. La droite de régression provient de l'ajustement linéaire des 9 derniers points (phase de transpiration purement cuticulaire).

fermeture des stomates est synchrone chez les feuilles de rosier excisées. $E_{\text {cut }}$ peut donc être calculé de la manière suivante (pour une feuille saturée en eau par convention):

$$
E_{\text {cut }}=(\Delta \mathrm{PE} / \Delta \mathrm{t}) / \mathrm{S}_{\mathrm{fol}}=(\mathrm{PFS}-\mathrm{PS}) \alpha / \mathrm{S}_{\mathrm{fol}}
$$

où $\mathrm{PE}$ correspond au poids d'eau, $\mathrm{S}_{\mathrm{fol}}$ à la surface foliaire, PFS au poids frais saturé et $\alpha$ à la pente de la droite de régression correspondant aux points de la "phase cuticulaire" de la courbe de transpiration $(\alpha=\Delta \log (\mathrm{PF}-\mathrm{PS}) / \Delta \mathrm{t})$.

En pratique, nous avons simplement associé une mesure du temps écoulé depuis le début de l'essai (PF $=$ PFS) à chacune des pesées, réalisées conjointement à la détermination du potentiel hydrique foliaire $(\psi)$. Cela nous a donc également permis d'associer a posteriori une valeur de $\psi$ à chaque point de ces courbes. Ainsi, le point correspondant à la fin de la "phase de fermeture stomatique " a pu être caractérisé pour chaque feuille étudiée par une valeur de $\psi: \psi^{\text {fer }}$.

Les données relatives à la transpiration des feuilles $\left(\psi^{\text {fer }}, \mathrm{E}_{\text {cut }}\right.$ ) ont été traitées par analyse de variance (2 serres, $2 \mathrm{EC}, 5$ périodes, 4 blocs). Les interactions entre serre, EC et saison ont été testées. Seules les probabilités supérieures à $95 \%$ ont été prises en considération (test $\mathrm{F}$ ).

\section{RÉSULTATS}

C'est en été, pendant la troisième période, qu'ont été observées les valeurs maximales 
et moyennes les plus basses de DSA (fig 2 et tableau I).

$\Psi^{\text {fer }}$ n'a pas été significativement affecté par l'EC, ni par l'HR; en revanche, $\Psi^{\text {fer }}$ a été affecté significativement par le temps (tableau II), remontant en été pour atteindre un maximum au début de l'automne et diminuer après (fig 3 ). Les stomates des feuilles de rosiers cultivés en hors-sol sont donc susceptibles de se fermer plus vite en été et au début de l'automne, ce qui est favorable au maintien de l'état hydrique des plantes mais défavorable à la photosynthèse. II faut noter que les variations de $\Psi^{\text {fer }}$ ne sont clairement pas synchrones avec celles du DSA : les valeurs de DSA les plus basses ont été observées pendant l'été (fig 2 et tableau I), alors que c'est au début de l'automne que $\Psi^{\text {fer }}$ a été le plus élevé (fig 3).

Les valeurs d' $E_{\text {cut }}$ sont comprises entre 0,2 et $0,810^{-3} \mathrm{~mol} \mathrm{~s}^{-1} \mathrm{~m}^{-2}$ (fig 4 ); elles apparaissent cohérentes avec d'autres valeurs observées, par exemple, par Grantz (1990) sur Glycine max Merr. II y a eu un effet significatif

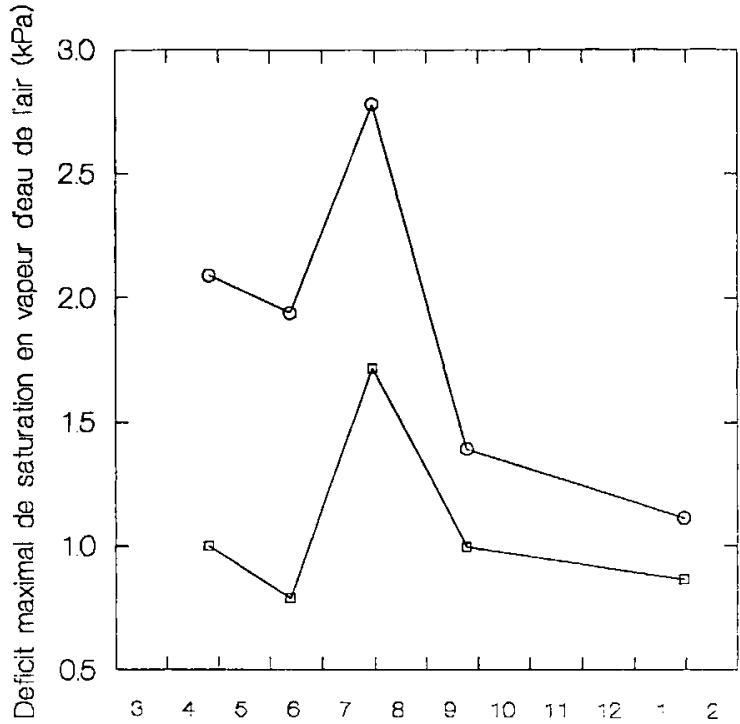

Mois

Fig 2. Évolution saisonnière du déficit maximal de saturation en vapeur d'eau de l'air. Les cercles correspondent au traitement à humidité relative (HR) basse et les carrés au traitement à HR élevée.

Tableau II. Résultats de l'analyse de variance de $\Psi^{\text {fer }}$ et $d^{\prime} E_{c u t}$ en fonction de la serre, de l'EC et de la saison.

\begin{tabular}{|c|c|c|c|c|c|}
\hline Source de variations & $D D L$ & $\begin{array}{c}\text { Somme } \\
\text { des carrés }\end{array}$ & $\begin{array}{l}\text { Carrés } \\
\text { moyens }\end{array}$ & $F$ & $P$ \\
\hline$\Psi^{f e r}$ & 1 & 0,186 & 0,186 & 3,292 & 0,1195 \\
\hline Serre & 1 & 0,039 & 0,039 & 0,450 & 0,5030 \\
\hline$E C$ & 4 & 19,845 & 4,961 & 57,297 & 0,0000 \\
\hline Période & 6 & 0,339 & 0,0565 & 0,649 & 0,6909 \\
\hline Bloc & 1 & 0,020 & 0,020 & 0,228 & 0,6339 \\
\hline Serre $\times$ période & 4 & 0,280 & 0,070 & 0,805 & 0,5233 \\
\hline $\mathrm{EC} \times$ période & 4 & 0,203 & 0,051 & 0,585 & 0,6740 \\
\hline Serre $\times E C \times$ période & 4 & 0,403 & 0,101 & 1,163 & 0,3282 \\
\hline Résidu & 198 & 17,144 & 0,087 & & \\
\hline Total & 223 & 38,459 & & & \\
\hline$E_{c u t}$ & 1 & 1,517 & 1,517 & 21,937 & 0,0034 \\
\hline Serre & 1 & 0,113 & 0,113 & 1,895 & 0,1705 \\
\hline $\mathrm{EC}$ & 4 & 3,922 & 0,981 & 16,478 & 0,0000 \\
\hline Période & 6 & 0,415 & 0,069 & 1,153 & 0,3343 \\
\hline Bloc & 1 & 0,026 & 0,026 & 0,437 & 0,5095 \\
\hline Serre $\times$ période & 4 & 0,755 & 0,189 & 3,173 & 0,0152 \\
\hline EC $\times$ période & 4 & 0,240 & 0,060 & 1,009 & 0,4045 \\
\hline Serre $\times E C \times$ période & 4 & 0,104 & 0,026 & 0,437 & 0,7818 \\
\hline Résidu & 168 & 9,998 & 0,060 & & \\
\hline Total & 193 & 17,09 & & & \\
\hline
\end{tabular}

$\Psi^{\text {fer }}$ : potentiel hydrique à la fermeture complète des stomates ; $E_{\text {cut }}$ : transpiration cuticulaire ; EC : électroconductivité de la solution nutritive. Les tests $F$ font intervenir le rapport entre le carré moyen des écarts du facteur étudié sur celui du résidu, à l'exception de l'effet serre (humidité relative) qui est le rapport sur le carré moyen des écarts des blocs dans la serre (bloc + bloc $\times$ serre). 


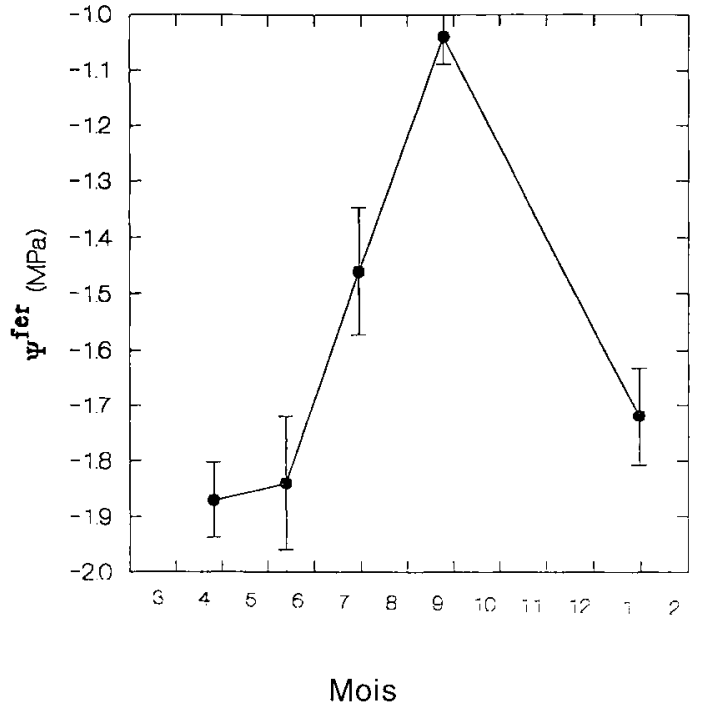

Fig 3. Évolution saisonnière du potentiel hydrique à la fermeture complète des stomates. Chaque cercle correspond à 48 feuilles, c'est-à-dire à toutes les données rassemblées des 4 traitements électroconductivité $\times$ humidité relative, aucun traitement n'ayant d'effet significatif. Les barres représentent les déviations standard.

de l'HR, mais pas de l'EC sur $E_{\text {cut }}$ (tableau II). Depuis le printemps jusqu'à l'été, $E_{\text {cut }}$ a été près de 2 fois plus élevé chez les feuilles du traitement à HR élevée que chez celles du traitement à HR basse (fig 4). On voit aussi que l'effet saisonnier sur $\mathrm{E}_{\text {cut }}$ a été

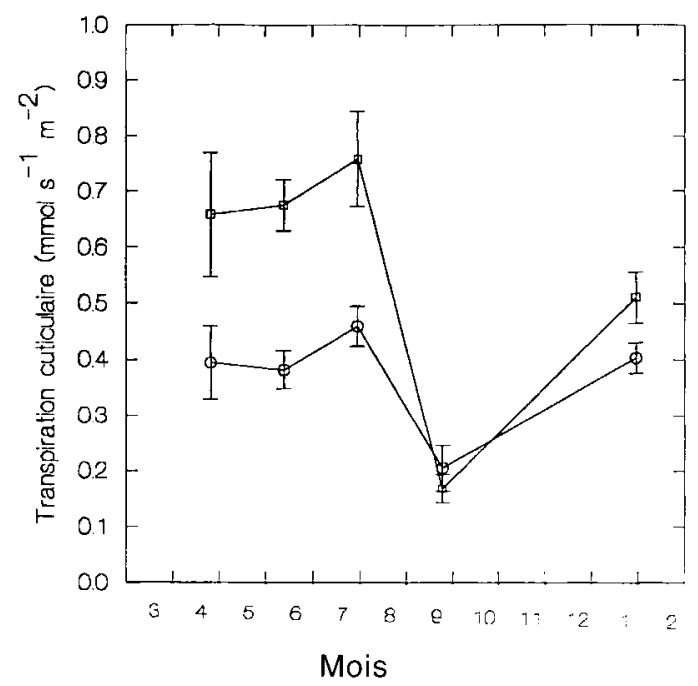

Fig 4. Évolution saisonnière du taux de transpiration cuticulaire. Les cercles correspondent au traitement à humidité relative (HR) basse et les carrés au traitement à HR élevée. Les barres représentent les déviations standard. plus marqué dans le traitement à HR élevée que dans le traitement à HR basse. La chute au début de l'automne d' $E_{\text {cut }}$ a été la plus forte pour les feuilles du traitement à HR élevée (diminution d'un facteur 3 environ). Dans tous les cas, $E_{\text {cut }}$ a chuté très fortement depuis l'été jusqu'au début de l'automne, pour augmenter à nouveau ensuite.

\section{DISCUSSION}

Nos observations sont cohérentes avec celles d'une diminution de la conductance stomatique faites chez Amygdalus communis $\mathrm{L}$, entre le mois de mai et le mois de juillet, par Torrecillas et al (1988). Une telle réduction de l'ouverture des stomates peut être attribuée à des changements ontogéniques (Solàrovà et Popsilovà, 1983). Cependant, dans le cas de rosiers cultivés en serre, il y a production, tout au long de l'année, de tiges et de feuilles nouvelles. Comme nous avons pris toutes les feuilles au même stade phénologique dans notre essai, on peut considérer que les variations du seuil de fermeture stomatique complète ne procèdent pas d'une évolution ontogénique.

Par ailleurs, Schoch (1978) a montré que la densité et l'indice stomatiques sont fortement dépendants des facteurs climatiques (intensité lumineuse, température et humidité relative de l'air). Mais, si la densité et l'indice stomatiques peuvent influer sur le taux de transpiration maximal (cuticulaire + stomatique avec les stomates complètement ouverts), en revanche, il n'y a pas de raison de penser que des différences de densité ou d'indice stomatiques peuvent se traduire par des différences de $\Psi^{\text {fer }}$.

II est couramment admis que l'adaptation à la sécheresse se traduit par une diminution du potentiel hydrique à la fermeture stomatique, c'est-à-dire par une augmentation de la quantité d'eau perdue requise avant la fermeture stomatique (Ludlow, 1980 ; Ludlow et al, 1985). L'ajustement osmotique est un important mécanisme d'adaptation aux déficits hydriques chez un grand nombre d'espèces (Turner et Jones, 1980). L'augmentation de la concentration intracellulaire en solutés permet de maintenir la turgescence requise pour la croissance cellulaire et l'ouverture stomatique à des niveaux de potentiel hydrique plus bas (Jones et Rawson, 1979). Mais lors- 
que les plantes ne sont pas soumises à des dessèchements progressifs, elles ne peuvent pas mettre en place de mécanismes d'adaptation à la sécheresse tels que l'ajustement osmotique (Ludlow et al, 1985). Or, nous avons montré, pour nos conditions expérimentales, que la disponibilité de l'eau n'a jamais été limitante dans le substrat, empêchant ainsi le développement progressif d'un déficit hydrique sévère comme on peut en observer en plein champ avec un sol qui se dessèche progressivement (Urban et al, 1994a). Enfin, ce n'est pas en été (lors de la troisième période), alors que la demande climatique était la plus forte, que $\Psi^{\text {fer }}$ a été le plus élevé (en valeur relative). Toutes ces observations tendent à exclure l'hypothèse selon laquelle c'est la dégradation estivale de l'état hydrique, liée à l'augmentation du rayonnement, de la température et du DSA, qui est à l'origine de l'augmentation de $\Psi^{\text {fer }}$.

En revanche, nous avons vu que le potentiel osmotique augmentait en été (en valeur relative) pour atteindre un maximum au début de l'automne, et diminuer à nouveau après (Urban et al, 1994b). Il y a un parallélisme remarquable entre les variations saisonnières de $\Psi^{\text {fer }}$, d'une part, et celles du potentiel osmotique, d'autre part. Il est donc permis de penser que c'est la même cause qui a été à l'origine de l'augmentation au début de l'automne, tout à la fois, de $\Psi^{\text {fer }}$ et du potentiel osmotique : en l'occurrence, la forte diminution, au début de l'automne, de la fraction d'eau non-osmotique (Urban et al, 1994b). Radin (1983) a montré qu'une diminution de la fraction d'eau non osmotique devait conduire à une augmentation du potentiel osmotique par simple effet de dilution, et inversement. Nous avons indiqué dans un article antérieur que les valeurs étonnamment élevées de la fraction d'eau non-osmotique, relevées par différents auteurs dans les feuilles de rosier, ne pouvaient pas s'expliquer uniquement par l'existence d'eau apoplastique mais aussi par celle d'eau associée à des macromolécules dans le symplasme (Urban et al, 1993). La diminution de la fraction d'eau non osmotique observée au début de l'automne dans des feuilles de rosier (Urban et al, 1994b) doit être rapprochée de la diminution des réserves d'amidon qui a été observée entre septembre et décembre chez le rosier par Zieslin et al (1975). Ackerson et Hebert (1981) ont observé que le volume osmotique tendait à diminuer en conséquence de l'accumulation d'amidon dans les feuilles de coton. Ils ont donc fait l'hypothèse que la concentration en solutés (et donc le potentiel osmotique) pouvaient varier avec les quantités d'amidon stockées. En résumé, nous émettons l'hypothèse que les variations saisonnières de $\Psi^{\text {fer }}$, observées dans les feuilles de rosier, résultent non pas de l'ontogenèse, ni de processus d'adaptation à la sécheresse, mais de variations saisonnières, a priori passives, de la concentration en solutés qui procèdent elles-mêmes de variations saisonnières des réserves.

Chez le sorgho, l'augmentation de l'épaisseur de cire épicuticulaire se traduit par une diminution de la transpiration cuticulaire (Blum, 1975). Premachandra et al (1992) ont montré sur feuilles de sorgho que la conductance cuticulaire était linéairement corrélée avec la charge de cire épicuticulaire. Cette augmentation de la charge de cire épicuticulaire réduit les pertes d'eau par transpiration (Jordan et al, 1984 ; Premachandra et al, 1992). En clair, il est probable que les variations de la transpiration cuticulaire que nous avons observées correspondent à des variations de la charge de cire épicuticulaire.

Nombreux ont été les auteurs ayant démontré l'influence de l'humidité relative sur la formation de la cuticule. Grout et Aston (1977) ont observé des difficultés de reprise à la sortie de culture in vitro chez les jeunes plants de chou-fleur régénérés à partir de méristèmes. Ces difficultés étaient liées à des pertes d'eau excessives dues à une formation insuffisante de cire épicuticulaire structurée. Sutter et Langhans (1979) ont montré que ce sont les niveaux d'HR élevés dans les bocaux de culture qui sont à l'origine du défaut de formation de cire épicuticulaire structurée des plants de chou-fleur issus de culture in vitro. Celle-ci se traduit par des pertes d'eau par transpiration beaucoup plus fortes que chez les plants issus de bouturage en serre. Après transfert en serre, les "vitroplants» forment des quantités croissantes de cire épicuticulaire structurée sous l'effet des niveaux d'HR relativement plus élevés. Capiellades et al (1990) ont noté sur plants de rosier, cultivés in vitro à $100 \%$ d'HR, des modifications anatomiques de la cire épicuticulaire et des cellules épidermiques, par rapport aux plants cultivés à $75 \%$ d'HR.

Nous n'avons aucune hypothèse satisfaisante pour expliquer les variations saisonnières d' $E_{\text {cut }}$ observées dans nos essais. Les 
variations saisonnières de DSA (ou d'HR) ne permettent pas de rendre compte de celles d'E $E_{\text {cut. }}$ En particulier, la chute d' $E_{\text {cut }}$ observée au début de l'automne (fig 4) n'apparaît pas comme la conséquence d'une augmentation du DSA, puisque celui-ci a, au contraire, diminué pendant la période correspondante (fig 2 et tableau I). Jordan et al (1983) et Saneoka et Ogata (1987) ont observé que la charge de cire épicuticulaire augmentait sous l'effet de la sécheresse chez le sorgho. Mais nos observations ont montré qu'il n'y a pas eu de situation de manque d'eau pour les plantes au début de l'automne (Urban et al, 1994a).

\section{CONCLUSION}

Nous venons de voir que le seuil de fermeture complète des stomates remonte du printemps jusqu'au début de l'automne. Si on admet que l'état hydrique commande l'ouverture des stomates, on peut donc être tenté de recourir à des techniques d'humidification de l'air en été pour améliorer l'état hydrique. Mais nous avons observé que le recours à l'humidification de l'air n'améliorait la conductance stomatique foliaire qu'en conditions d'arrosage imparfaites (Urban et Langelez, 1992). Il est probable également que l'effet positif de la nébulisation fine sur la transpiration cuticulaire des feuilles de rosier représente un inconvénient pour la phase de post-récolte.

Les variations saisonnières du seuil de fermeture stomatique des feuilles de rosiers cultivés en hors-sol sous serre ne correspondent pas à une variation saisonnières de la demande climatique. Elles ne semblent pas correspondre non plus à une évolution ontogénique des feuilles. II serait intéressant de vérifier plus directement l'hypothèse selon laquelle ce sont les variations saisonnières de l'état des réserves qui commandent celles du seuil de fermeture stomatique.

Par ailleurs, il semblerait utile de vérifier que le comportement de feuilles soustraites aux corrélations entre organes est similaire à celui de feuilles encore attachées à la tige florale récoltée et d'évaluer plus précisément l'influence des variations saisonnières du seuil de fermeture stomatique et de la transpiration cuticulaire sur la tenue en vase des roses.

\section{REMERCIEMENTS}

Les auteurs tiennent à exprimer leurs remerciements à $S$ Adamowicz, $R$ Brun, $A$ Coudret et $E$ Dreyer pour leur aide et leurs commentaires précieux.

\section{RÉFÉRENCES}

Ackerson RC, Hebert RR (1981) Osmoregulation in cotton in response to water. I. Alterations in photosynthesis, leaf conductance, translocation and ultrastructure. Plant Physiol 67, 484-4884

Blum A (1975) Effet of the Bm gene on epicuticula wax and the water relations of Sorghum bicolor. Isr $J$ Bot 24, 50

Brun R, Tramier PH (1988) Culture du rosier sur laine de roche. Rev Hortic 289, 43-51

Brun R, Paris B, Urban L, Pyrrha P (1994) Management of the fertilizing irrigation of rose plants grown in greenhouses on rockwoll. Acta Hortic (sous presse)

Capellades M, Fontarnau R, Carulla C, Debergh $P$ (1990) Environmental influences on anatomy of stomata and epidermal cells in tissue-cultured Rosa multiflora. J Am Soc Hortic Sci 115, 141-145

Coker T, Mayak S, Thompson JE (1985) Effect of water stress on ethylene production and on membrane microviscosity in carnation flowers. Sci Hortic 27, 317-324

De Villèle O (1972) Besoins en eau des cultures sous serres. Essai de conduite des arrosages en fonction de l'ensoleillement. Acta Hortic 35 123-130

Fabret C (1993) Influence des relations hydriques et de l'état des réserves en sucres sur la qualité des roses. Mémoire de fin d'études de I'ENITA de Clermont-Ferrand, $48 \mathrm{p}$

Grantz DA (1990) Plant response to atmospheric humidity. Plant Cell Env 13, 667-679.

Grout BWW, Aston MJ (1977) Transplanting of cauliflower plants regenerated from meristem culture. II. Carbon dioxide fixation and development of photosynthetic ability. Hortic Res 17 , 65-71

Hygen G (1951) Studies in plant transpiration. I, Physiol Plant 4, 57-183

Hygen G (1953) Studies in plant transpiration. II, Physiol Plant 6, 106-133

Jones MM, Rawson HM (1979) Influence of rate of development of leaf water deficits upon photosynthesis, leaf conductance, water use ef- 
ficiency and osmotic potential in sorghum. Physiol Plant 45, 103-111

Jordan WR, Monk RL, Miller FR, Rosenow DT, Clark LE, Shouse PJ (1983) Environmental physiology of sorghum. I. Environmental and genetic control of epicuticular wax load. Crop Sci 23, 552-558

Jordan WR, Shouse PJ, Blum A, Miller FR, Monk RL (1984) Environmental physiology of sorghum. II. Epicuticular wax load and cuticular transpiration. Crop Sci 24, 1168-1173

Ludlow MM (1980) Adaptative significance of stomatal responses to water stress. In: Adaptation of plants to water and high temperature stress, (NC Turner, PJ Kramer, eds). Wiley and sons, New York, 123-138

Ludlow MM, Fisher MJ, Wilson JR (1985) Stomatal adjustment to water deficits in three tropical grasses and a tropical legume grown in controlled conditions and in the field. Aust $J$ Plant Physiol 12, 131-149

Masson B, Paulin A (1980) Influence of temporary water stress on the water balance of cut carnations. CR Acad Agric Fr 66, 1498-1506

Paulin A, Kerhardy F, Maestri B (1985) Effect of drought and prolonged refrigeration on senescence in cut carnation (Dianthus Caryophyllus L). Physiol Plant 64, 536-540

Paull RE, Goo TTC (1985) Ethylene and water stress in the senescence of cut anthurium flowers. J Am Soc Hortic Sci 110, 84-88

Premachandra GS, Saneoka G, Fujita K, Ogata $S$ (1992) Leaf water relations, osmotic adjustement cell membrane stability, epicuticular wax load and growth as affected by increasing water deficits in sorghum. J Exp Bot 257, 15691576

Radin JW (1983) Physiological consequences of cellular water deficits: osmotic adjustment. In: Limitations to efficient water use in crop production (J Grace, ED Ford, PG Jarvis, eds) American Society of Agronomy, Madison, 267275

Saneoka H, Ogata S (1987) Relationship between water use efficiency and cuticular wax deposition in warm season forage crops grown under water deficit conditions. Soil Sci Plant Nut 33, $439-448$

Schoch PG (1978) Différenciation numérique des stomates du Vigna sinensis $L$ et de quelques autres espèces. Thèse de Doctorat d'État èsSciences, Université d'Aix-Marseille II, 227 p

Solarova J, Popsilova J (1983) Photosynthetic characteristics during ontogenesis of leaves 8 . Stomatal diffuse conductance and stomatal reactivity Photosynthetica 17, 101-151

Sutter E, Langhans RW (1979) Epicuticular wax formation on carnation plantlets regenerated from shoot tip culture. J Am Soc Hortic Sci 104, 493-496

Tardieu F, Davies WJ (1992) Stomatal response to abscisic acid is a function of current plant water status. Plant Physiol 98, 540-545

Torrecillas A, Ruiz-Sanchez M C, Del Amor F, Leon A (1988) Seasonal variations on water relations of Amygdalus communis $L$ under drip irrigated and non-irrigated conditions. Plant Soil $106,215-220$

Turner NC, Jones MM (1980) Turgor maintenance by osmotic adjustement. In: Adaptation of plants to water and high temperature stress (NC Turner, PJ Kramer, eds). Wiley-Interscience, New York, 343-372

Urban L, Langelez I (1992) Effect of high pressure mist on leaf water potential, leaf diffusive conductance, $\mathrm{CO}_{2}$ fixation and production of 'Sonia' rose plants grown in rockwool. Sci Hortic 50, 229-244

Urban L, Jaffrin A, Chraibi A (1993) Analysis of pressure-volume curves of Rosa hybrida, cV Sonia leaves. J Exp Bot 44, 605-613

Urban L, Brun R, Pyrrha P (1994a) Water relations of leaves of Sonia rose plants grown in rockwool at two electrical conductivities under controlled and uncontrolled relative humidity. Hort Sci (sous presse)

Urban L, Brun R, Pyrrha P (1994b) Seasonal variations of water relations in roses. Biol Plant (sous presse)

Van Gardinen PR, Grace J (1992) Vapour pressure deficit response of cuticular conductance in intact leaves of Fagus sylvatica L. J Exp Bot 255, 293-1299

Zieslin N, Hurwitz A, Halevy AH (1975) Flower production and the accumulation and distribution of carbohydrates in different parts of 'Baccara' rose plants as influenced by various pruning and pinching treatments. J Hortic Sci 50, 339348 